\title{
ODINOFAGIA Y CERVICOBRAQUIALGIA EN SÍNDROME DE EAGLE. DESCRIPCIÓN DE UN CASO
}

\section{Odynophagia and cervicobrachialgia in Eagle's Syndrome. A case report}

\author{
Elena SÁNCHEZ-LEGAZA ${ }^{1}$; José Luis REPETTO-LÓPEZ²; Regla GALLEGO-GALLEGOS ${ }^{3}$ \\ Hospital de Algeciras. ${ }^{1}$ Servicio de Otorrinolaringología. ${ }^{2}$ Servicio de Traumatología. ${ }^{3}$ Servicio de Aparato \\ Digestivo. Cádiz. España. \\ Correspondencia: manpro1910@hotmail.com
}

Fecha de recepción: 18 de agosto de 2016

Fecha de aceptación: 26 de septiembre de 2016

Fecha de publicación: 28 de septiembre de 2016

Fecha de publicación del fascículo: 1 de marzo de 2017

Conflicto de intereses: Los autores declaran no tener conflictos de intereses

Imágenes: Los autores declaran haber obtenido las imágenes con el permiso de los pacientes

Política de derechos y autoarchivo: se permite el autoarchivo de la versión post-print (SHERPA/RoMEO)

Licencia CC BY-NC-ND. Licencia Creative Commons Atribución-NoComercial-SinDerivar 4.0 Internacional

๑) Universidad de Salamanca. Su comercialización está sujeta al permiso del editor

\begin{abstract}
RESUMEN Introducción: El síndrome de Eagle es una rara entidad, en la que el proceso estiloideo o ligamento estilohioideo está elongado y/o calcificado. Descripción: Se presenta el caso de un varón con odinofagia y dolor cervical irradiado a brazos con síndrome de Eagle. Discusión: EI síndrome de Eagle suele ser asintomático y unilateral, aunque puede ser bilateral y dar síntomas muy variados, dependiendo de la estructura que comprima como disfagia, odinofagia, sensación de cuerpo extraño faríngeo, cefalea y otalgia. El diagnóstico se basa en la exploración clínica confirmada con pruebas de imagen. Conclusiones: El síndrome de Eagle debe incluirse en el diagnóstico diferencial de algias cervicofaciales y patología de la articulación temporomandibular.

PALABRAS CLAVE síndrome de Eagle; proceso estiloideo elongado; odinofagia

SUMMARY Introduction: Eagle syndrome is a rare entity, in which the styloid process or stylohyoid liga-
ment is elongated and / or calcified. Description: We present the case of a male patient with
odynophagia and cervical pain radiating to both arms. Discussion: Usually it is asymptomatic
and unilateral, although it may be bilateral and give different symptoms, depending on the
compressed structure, such as dysphagia, sore throat, pharyngeal foreign body sensation,
headache, earache. The diagnosis is based on a good clinical examination confirmed with
imaging studies. Conclusions: Eagle's syndrome should be included in the differential diag-
nosis of cervico-facial pain and pathology of the temporomandibular joint.
\end{abstract}

KEYWORDS

Eagle's syndrome; elongated styloid process; odynophagia 


\section{INTRODUCCIÓN}

El síndrome de Eagle (SE) es una complicación rara de la elongación del proceso estilohioideo con más de $25 \mathrm{~mm}$ de longitud o calcificación del ligamento estilohioideo, y en ocasiones del asta menor del hioides, descrito por Eagle en 1937 [1]. Suele cursar de forma asintomática, descubriéndose como un hallazgo radiológico. Afecta al $4 \%$ de la población, aunque en el $4 \%$ al $7 \%$ aparece clínica como odinofagia, sensación de cuerpo extraño, disfagia, dolor faríngeo o cervical -al abrir la boca, al hablar o con movimientos del cueIlo-, otalgia refleja y puede confundirse con un algia facial [2].

Eagle postuló dos tipos: el clásico, caracterizado por dolor espástico y persistente en faringe, sialorrea y náuseas y el síndrome estilocarotídeo, secundario a irritación de las fibras simpáticas pericarotídeas, que cursa con dolor cervical al giro de la cabeza, migraña y síntomas neurológicos por irritación del plexo simpático [1]. En ocasiones, se afectan los pares craneales V, VII, IX, X, XI y XII y la vena yugular interna, manifestándose como neuralgia y parálisis del nervio glosofaríngeo, síndrome de Horner y parálisis facial. Camarda distinguió tres entidades clínicas distintas: la primera, el SE propiamente dicho, que requiere la presencia de una cirugía a nivel cervical o trauma previos, junto a dolor a la palpación clínica del proceso estiloides elongado, confirmado con un diagnóstico de imagen; la segunda, el síndrome estilohioideo, en el que el paciente de edad adulta relata sintomatología confirmada con pruebas de imagen sin estar sometido a cirugía previa; y la tercera, el síndrome pseudoestilohioideo, en el que el paciente describe los síntomas clásicos del SE, pero no existe una presencia evidente, clínica ni radiográfica, de elongación u osificación del proceso estiloides, ni cirugía previa o trauma, siendo los síntomas consecuencia del envejecimiento, ya que debido a la pérdida de elasticidad de los tejidos, es más frecuente la existencia de tendinitis o reacciones inflamatorias que causan dolor en territorio glosofaríngeo [3].

El diagnóstico se basa en la clínica y la palpación digital sobre la fosa amigdalina de una prominencia dura y dolorosa. Se confirma con una radiografía anteroposterior modificada (vista de Towne), radiografía lateral de cráneo, ortopantografía, TAC y angiografía (para descartar la afectación de la arteria carótida).

\section{DESCRIPCIÓN}

Paciente varón de 49 años, sin antecedentes quirúrgicos y con cardiopatía hipertensiva que acudió a consultas externas de traumatología por dolor progresivo difuso en cuello y pesadez en brazos de 6 meses de evolución, que se intensificaba con esfuerzos, pero que no cedía con analgésicos no esteroideos. Además, refería odinofagia y sensación de cuerpo extraño faríngeo. En la exploración refería dolor cervical irradiado a brazos sin distribución radicular de 2 a 5 minutos. Exploración neurológica, electroneurografía y electromiografía, ecografía Doppler de troncos supraaórticos normales. En la radiografía lateral de cuello presentaba un proceso estilohioideo bilateral largo de $7 \mathrm{~mm}$ (Figura 1). Fue remitido a consulta externa de otorrinolaringología para descartar síndrome de Eagle. La rinofibrofaringoscopia fue normal con palpación cervical normal. La ortopantografía y TAC cervical que mostraban elongación de apófisis estiloides y calcificación bilateral de los ligamentos estiloideos, que confirmaron el diagnóstico de SE (Figuras 2 y 3 ). Se informó la posibilidad de tratamiento médico y quirúrgico del proceso, y el paciente eligió revisiones periódicas.

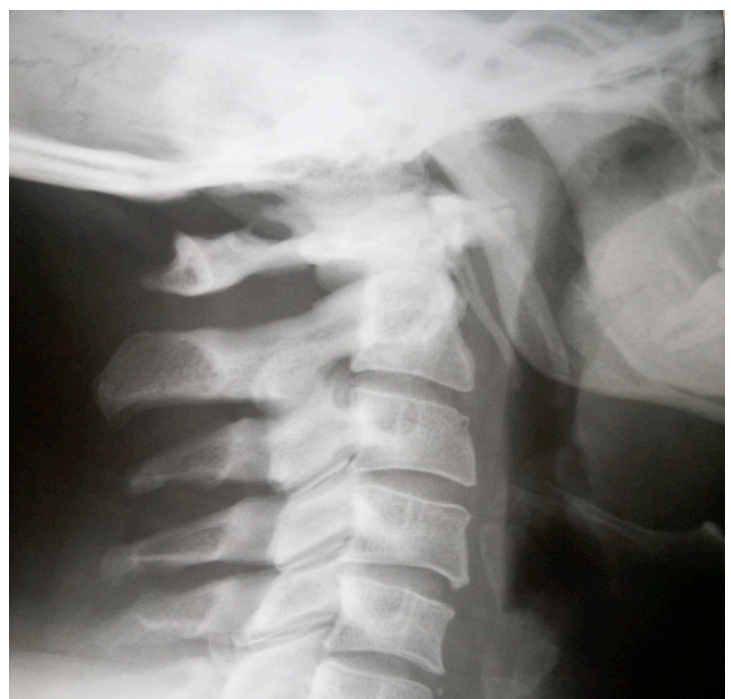

Figura 1. Radiografía lateral cervical con elongación y osificación de ambos procesos estilohioideos.

\section{DISCUSIÓN}

EI SE es una rara entidad secundaria a la elongación o calcificación del proceso estiloides. Su etiopatogenia es desconocida, aunque Murtagh (2001) presentó tres teorías: la 
primera, es la elongación del proceso estilohioideo, debido a la persistencia del cartílago precursor; la segunda, es la calcificación del ligamento estilohioideo por un proceso desconocido [4]; y la tercera, por crecimiento del tejido óseo de la inserción del ligamento estilohiodeo.

Steinmann (1968) propuso tres mecanismos: hiperplasia reactiva desencadenada por un traumatismo, metaplasia reactiva o cicatrización anómala tras un trauma, o una variación anatómica sin trauma previo. Camarda (1989) añadió un cuarto mecanismo, un anormal desarrollo del envejecimiento sin osificación previa [5]. El caso presentado puede clasificarse como un síndrome estilohioideo.

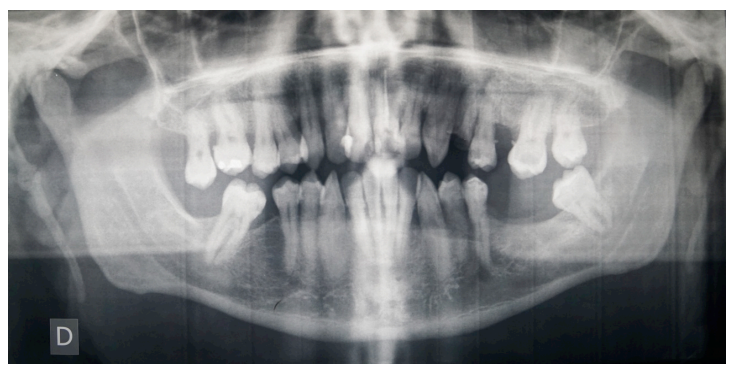

Figura 2. Ortopantografía.

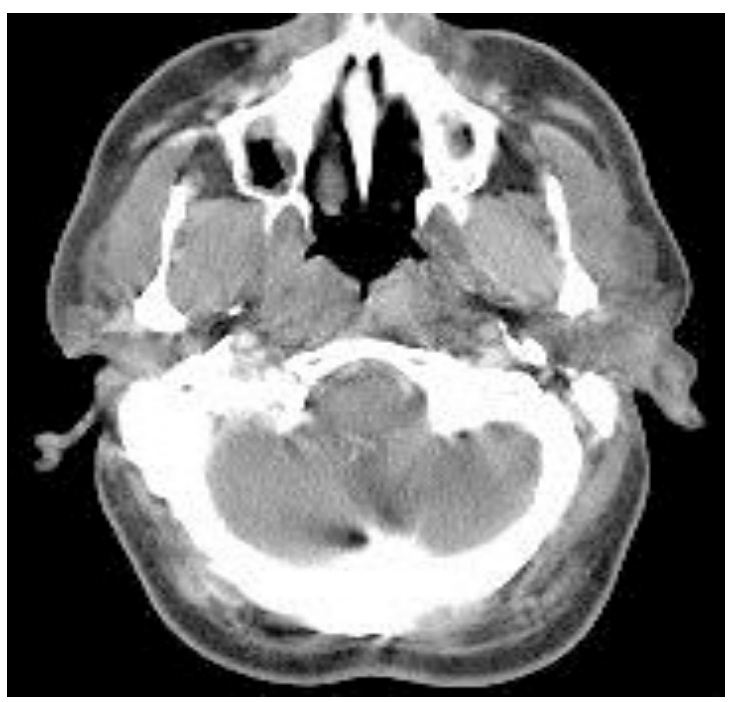

Figura 3. TAC craneal confirman el alargamiento de los ligamentos estilohioideos.

Eagle comentó que un trauma quirúrgico previo, como amigdalectomía o una irritación crónica del ligamento estilomandibular, podía causar osteítis, tendinitis o periostitis, condu- ciendo a una hiperplasia osificante reactiva del proceso estiloides, y otros autores creían que era debido a la presencia de elementos mesenquimales o desordenes endocrinos. El proceso estiloides deriva del cartílago de Reichert del segundo arco branquial, que persiste como una estructura originada de la porción petrosa del temporal, entre la arteria carótida interna y externa, adyacente a los nervios glosofaríngeo y vago. La longitud media del proceso estiloides es de $3 \mathrm{~cm}(1,52$ a $4,77 \mathrm{~cm}$ ), y dicha longitud no se relaciona con la severidad del dolor [6].

Suele ser más frecuente en mujeres de la tercera y cuarta década de la vida. Suele causar dolor sordo o recurrente de garganta, sensación de cuerpo extraño faríngeo con otalgia refleja, odinofagia, disfagia [7] o dolor en el área del nervio glosofaríngeo, y más raramente dolor cervicofacial con limitación de movimientos cervicales, sobre todo con la deglución, apertura de la boca y los movimientos del cuello. Se han descrito parestesias en hemilengua - por su proximidad al nervio lingual- [6], paresia del nervio hipogloso, síndrome de Horner [8] o ictus de repetición ocasionado por la compresión de la arteria carótida interna por el proceso estiloides [9]. Se diagnostica ante un dolor cervicofacial uni o bilateral atípico que no responde a analgésicos habituales, junto con la exploración clínica -la palpación en la fosa amigdalina del pilar anterior de una cuerda o punta ósea que produce o exacerba el dolor- y radiológica -siendo la radiografía panorámica y la lateral de cuello las utilizadas, pero su grado de distorsión de hasta el $37 \%$ y la interposición de estructuras lleva a errores, por lo que se aconseja TAC con la boca abierta, y últimamente TAC con reconstrucciones $3 \mathrm{D}$ para su confirmación- [10, 11]. Debe hacerse diagnóstico diferencial de dolor atípico en la región de cabeza y cuello con: algia craneofaringofacial, neuralgia del trigémino, neuralgia esfenopalatina y del glosofaríngeo, patología de articulación temporomandibular, síndrome de Sluder, migraña, y arteritis de la arteria temporal [12]. A veces el diagnóstico del dolor orofacial inespecífico puede ser complicado, requiriendo un abordaje multidisciplinar [13].

El tratamiento suele ser médico en casos leves y moderados -analgésicos, anticonvulsivantes, antidepresivos, e infiltración transfaríngea con anestésicos locales o esteroides en la celda amigdalina, o carbamazepina oral-, pero los resultados a largo plazo no

(c) Ediciones Universidad de Salamanca / CC BY-NC-ND [67] Rev. ORL, 2017, 8, 1, pp. 65-68 
son satisfactorios. En los casos sintomáticos severos con dolor intenso y refractario, se propone tratamiento quirúrgico -resección parcial, mediante abordaje intra o extraoral[14]. Algunos autores, proponen el abordaje transoral, por la facilidad en el abordaje con menor tiempo quirúrgico, no dejar cicatrices, y la ausencia de complicaciones infecciosas posoperatorias, reduciendo el periodo de hospitalización [15], aconsejándose en aquellos casos en los que se identifica la apófisis estiloides por palpación intraoral. Sin embargo, la vía transcervical permite mejor acceso con el uso de instrumental piezoeléctrico, por su corte selectivo y preciso, resultando ser segura y efectiva, a pesar del riesgo de lesionar la rama mandibular marginal del facial $[16,17]$.

\section{CONCLUSIONES}

El síndrome de Eagle es una causa infrecuente de odinofagia que debe formar parte de su diagnóstico diferencial y de otras algias cervicofaciales.

\section{BIBLIOGRAFÍA}

1. Eagle W. Elongated styloid process: report of two cases. Arch Otolaryngol. 1937;25:584-7.

2. Gelabert-González $M$, García-Allut A. Síndrome de Eagle: Una causa poco frecuente de cervicalgia. Neurocirugía. 2008;19:254-6.

3. Cantín, L.M, Suazo Galdames, I. Síndrome de Eagle o síndrome estilohioideo? Neurologia. 2011;26:254-5.

4. Murtagh RD, Caracciolo JT, Fernández G. CT findings associated with Eagle syndrome. AJNR Am J Neuroradiol. 2001;22:1401-2.

5. Moon C, Lee B, Kwon Y, Chol B, Lee J, Lee H. Eagle's syndrome: a case a report. J Korean Assoc Oral Maxillofac Surg. 2014;40:43-7.

6. Kiralj A, Illić M, Pejaković B, Markov B, Mijatov S, Mijatov I. Eagle's syndrome. A report of two cases. Vojnosanit Pregl 2015; 72(5):548-62.

7. Dong Z, Bao H, Zhang L, Hua Z. Eagle's syndrome associated with lingual nerve paresthesia: a case report. J Oral Maxillofac Surg. 2014;72:886.e1-4.
8. Madden LL, Gross RD, Smith LJ. Bilateral Eagle syndrome causing dysphagia. Ear Nose Throat J. 2015;94:69-70.

9. Bensoussan Y, Letourneau-Guillon L, Ayad T. Atypical presentation of Eagle syndrome with hypoglossal nerve palsy and Horner syndrome. Head Neck. 2014;36:E136-8.

10. Kent DT, Rath TJ, Synderman C. Conventional and 3-Dimensional Computerized Tomography in Eagle's Syndrome, Glosopharyngeal Neuralgia and Asyntmatic Controls. Otolaryngol Head Neck Surg. 2015;153:41-7.

11. Sanz Gonzalo JJ, Maiz Cal J, Lao Luque $\mathrm{X}$, Luzardo C. Síndrome de Eagle: ictus de repetición como presentación clínica; calcificación estilohioidea completa. Acta Otorrinolaringol Esp. 2010;61:233-5.

12. Ferreira PC, Mendanha M, Frada $\mathrm{T}$, Carvalho J, Silva A, Amarante J. Eagle syndrome. J Craniofac Surg. 2014;25:e84-6.

13. Kamal A, Nazir R, Usman M, Salam B, Sana F. Eagle syndrome; radiological evaluation and management. JPMA. 2014;64:1315-7.

14. Martins WD, Ribas MdeO, Bisinelli J, Franca BH, Martins G. Eagle's syndrome: treatment by intraoral bilateral resection of the ossified stylohyoid ligament. A review and report of two cases. Cranio 2013;31:226-31.

15. Müderris T, Bercin S, Sevil E, Beton S, Kiris M. Surgical management of elongated styloid process: intraoral or transcervical. Eur Arch Otorhinolaryngol. 2014;271:1709-13.

16. Mareque Bueno J, Hernández Alfaro F, Biosca de Tejada MJ, Coll Anglada M, Arenaz J. Abordaje intraoral en el síndrome de Eagle. Presentación de un caso clínico. Rev Esp Cir Oral Maxilofac. 2011;33:157-61.

17. Bertossi D, Albanese M, Chiarini L, Corega C, Mortellaro C, Nocini P. Eagle syndrome surgical treatment with piezosurgery. J Craniofac Surg. 2014;25:811-3. 